\title{
On Iteration Method to The Solution of More General Volterra Integral Equation in Two Variables and a Data Dependence Result
}

\author{
Samet MALDAR ${ }^{1 *}$ iD \\ ${ }^{1}$ Aksaray University, Faculty of Science and Letters, Department of Mathematics, Aksaray, Turkey \\ *mmaldar@aksaray.edu.tr \\ *Orcid: 0000-0002-2083-899X
}

Received: 7 December 2020

Accepted: 16 September 2021

DOI: $10.18466 /$ cbayarfbe. 837062

\begin{abstract}
Fixed point theory is one of the most important theories and has been studied extensively by researchers in many disciplines. One of these studies is its application to integral equations. In this work, we have shown that the iteration method given in [30] converges to the solution of the more general Volterra integral equation in two variables by using Bielecki's norm. Also, a data dependence result for the solution of this integral equation has been proven.
\end{abstract}

Keywords: convergence, data dependence, integral equation, iteration method Mathematics Subject Classification: 47H06; 47H10, 54H25.

\section{Introduction}

Various problems in nature can be expressed in nonlinear equations. Reaching the solution of nonlinear equations is significant for many disciplines. There are many methods to obtain this solution in mathematics. One of them is the fixed-point theory. One of the study areas of nonlinear analysis is integral equations and one way to show the existence and uniqueness of the solutions of integral equations is iteration methods in fixed point theory. Iteration methods have become an intriguing method for solving nonlinear equations. In this context, a large literature has emerged with the definition of new iteration methods (see [1-5]). Fixed point iteration methods have been studied by many researchers to solve integral equations (see [8-29]). The basic approach in this method is to construct iteration methods by including the integral equations in an operator classification under certain conditions and to determine the appropriate conditions for the sequence obtained from this iteration to converge to the fixed point of the operator, in other words, to the solution of the integral equation. In this regard, Lungu and Rus [6] have proved that defined Volterra-Fredholm integral equation (1.1) under the conditions given below (c1-c6) has only unique solution.

In more general form, Volterra-Fredholm integral equation [6] in two variables can be seen as $g(x, y)=f(x, y,(h(g))(x, y)))+$

$\int_{0}^{x} \int_{0}^{y} K(x, y, s, t, g(s, t)) d s d t \quad x, y \in \mathbb{R}_{+}$.

Let $(E,\|\cdot\|)$ be a Banach space. Let $K \in C\left(\mathbb{R}^{4}{ }_{+} \times\right.$ $E, E)$ be class of continuous functions. Bielecki's norm [7] on $X_{\tau}$ defined as

$$
\|g\|_{\tau}=\sup _{x, y \in \mathbb{R}_{+}}(\|g(x, y)\|) e^{-\tau(x+y)}
$$

such that

$X_{\tau}=\left\{g \in C\left(\mathbb{R}^{2}{ }_{+}, E\right)|\exists M(g)>0:| g(x, y) \mid \leq\right.$ $\left.e^{-\tau(x+y)} \leq M(g)\right\}$

for $\tau>0$.

It is clear that $\left(X_{\tau},\|\cdot\|_{\tau}\right)$ is a Banach space.

Teorem 1.1 Let us assume that the following conditions are satisfied:

(c1) $f \in C\left(\mathbb{R}^{2}{ }_{+} \times E, E\right), K \in C\left(\mathbb{R}^{4}{ }_{+} \times E, E\right)$,

(c2) $\forall x, y \in \mathbb{R}_{+}, \forall u, v \in X_{\tau}, h: X_{\tau} \rightarrow X_{\tau}$ such that $\left.\left.\exists L_{h}>0: \|(h(u))(x, y)\right)-(h(v))(x, y)\right) \|$ $\leq L_{h}\|u-v\|_{\tau} e^{\tau(x+y)}$,

(c3) $\forall x, y \in \mathbb{R}_{+}, \forall w_{1}, w_{2} \in E$;

$\exists L_{f}>0:\left\|f\left(x, y, w_{1}\right)-f\left(x, y, w_{2}\right)\right\| \leq L_{f}\left\|w_{1}-w_{2}\right\|$, 
(c4) $\forall x, y, s, t \in \mathbb{R}_{+}, \forall w_{1}, w_{2} \in E$;

$\exists L_{K}(x, y, s, t)>0:\left\|K\left(x, y, s, t, w_{1}\right)-K\left(x, y, s, t, w_{2}\right)\right\|$ $\leq L_{K}(x, y, s, t)\left\|w_{1}-w_{2}\right\|$,

(c5) $\forall x, y \in \mathbb{R}_{+}$,

$L_{K} \in C\left(\mathbb{R}^{4}{ }_{+}, \mathbb{R}_{+}\right)$and $\int_{0}^{x} \int_{0}^{y} L_{K}(x, y, s, t) e^{\tau(s+t)} d s d t \leq$ $L e^{\tau(x+y)}$,

(c6) $L_{h} L_{f}+L<1$.

Then (1.1) has a unique solution [6].

The following iteration method has defined in [30].

$$
\left\{\begin{array}{c}
x_{0} \in X, \\
x_{n+1}=T\left(\frac{\left(1-\alpha_{n}\right)}{k} T x_{n}+\left(1-\frac{\left(1-\alpha_{n}\right)}{k}\right) T y_{n}\right) \\
y_{n}=T\left(\frac{\left(1-\beta_{n}\right)}{k} x_{n}+\left(1-\frac{\left(1-\beta_{n}\right)}{k}\right) T x_{n}\right)
\end{array}\right.
$$

where $\left\{\alpha_{n}\right\}_{n=1}^{\infty}$ and $\left\{\beta_{n}\right\}_{n=1}^{\infty}$ are real sequences in $[0,1]$, $k, n \in \mathbb{N}$ and $T$ is any self-operator. The iteration method (1.4) can be demonstrated as follows:

$$
\left\{\begin{array}{c}
x_{0} \in X \\
x_{n+1}=T u_{n} \\
u_{n}=\frac{\left(1-\alpha_{n}\right)}{k} T x_{n}+\left(1-\frac{\left(1-\alpha_{n}\right)}{k}\right) T y_{n} \\
y_{n}=T v_{n} \\
v_{n}=\frac{\left(1-\beta_{n}\right)}{k} x_{n}+\left(1-\frac{\left(1-\beta_{n}\right)}{k}\right) T x_{n} .
\end{array}\right.
$$

Definition 1.2 Let $X$ be a Banach space and $C$ be a nonempty, closed, convex subset of $X$. Let $S, T: C \rightarrow C$ be two mappings. We say that the $S$ is an approximate mapping pair of $T$ if for all $x \in C$ and for a fixed $\epsilon \geq 0$, we have $\|T x-\mathrm{S} x\| \leq \epsilon[31]$.

Lemma 1.3 Let $\left\{\sigma_{n}\right\}_{n=0}^{\infty}$ be nonnegative real sequence. Assume that there exists $n_{0} \in \mathbb{N}$, such that for all the $n \geq n_{0}$ one has the inequality

$\sigma_{n+1} \leq\left(1-\lambda_{n}\right) \sigma_{n}+\lambda_{n} m_{n}$

where $\lambda_{n} \in(0,1)$ for all $n \in \mathbb{N}, \sum_{n=0}^{\infty} \lambda_{n}=\infty$, and $m_{n} \geq 0$. Then the following inequality holds:

$0 \leq \lim _{n \rightarrow \infty} \sup \sigma_{n} \leq \lim _{n \rightarrow \infty} \sup m_{n}[31]$

Consider the equation (1.1), we have $T: X_{\tau} \rightarrow X_{\tau}$ defined by

$\left.T\left(x_{n}(x, y)\right)=f\left(x, y,\left(h\left(x_{n}\right)\right)(x, y)\right)\right)$

$+\int_{0}^{x} \int_{0}^{y} K\left(x, y, s, t, x_{n}(s, t)\right) d s d t \quad x, y \in \mathbb{R}_{+}$.

In this work, we have shown that the iteration method (1.5) converges to the solution of Volterra-Fredholm integral equation given by (1.1) in Banach space $X$ for the initial point $x_{0} \in X$. Also, we have obtained the data dependence result for the solution of equation (1.1) under conditions in Theorem 1.1.

\section{Main Results}

Teorem 2.1 Let $\left\{\alpha_{n}\right\}_{n=1}^{\infty}$ and $\left\{\beta_{n}\right\}_{n=1}^{\infty}$ are real sequences in $[0,1]$. Then under the assumptions of Theorem 1.1, the equation (1.1) has a unique solution, say $x_{p}$, and the iteration method (1.5) is convergent strongly to $x_{p}$.

Proof Let $\left\{x_{n}\right\}_{n=1}^{\infty}$ be an iterative sequence generated by iteration method (1.5) as follows:

$\left.T\left(x_{n}(x, y)\right)=f\left(x, y,\left(h\left(x_{n}\right)\right)(x, y)\right)\right)+$ $\int_{0}^{x} \int_{0}^{y} K\left(x, y, s, t, x_{n}(s, t)\right) d s d t \quad x, y \in \mathbb{R}_{+}$

where $T: X_{\tau} \rightarrow X_{\tau}$

We will show that $x_{n} \rightarrow x_{p}$ as $n \rightarrow \infty$. From (1.1), iteration method (1.5), and the assumptions (c1)-(c6) of Theorem 1.1, we obtain

$\left\|x_{n+1}-x_{p}\right\|_{\tau}=\left\|T u_{n}-T x_{p}\right\|_{\tau}=$
$\sup _{x, y \in \mathbb{R}_{+}}\left(\left\|T u_{n}(x, y)-T x_{p}(x, y)\right\| e^{-\tau(x+y)}\right)$

and

$\left\|T u_{n}(x, y)-T x_{p}(x, y)\right\|=$

$\left.\| f\left(x, y,\left(h\left(u_{n}\right)\right)(x, y)\right)\right)$

$+\int_{0}^{x} \int_{0}^{y} K\left(x, y, s, t, u_{n}(s, t)\right) d s d t$

$-f\left(x, y, h\left(x_{p}(x, y)\right)\right)$

$\left\|-\int_{0}^{x} \int_{0}^{y} K\left(x, y, s, t, x_{p}(s, t)\right) d s d t\right\|$

$\leq \| f\left(x, y,\left(h\left(u_{n}\right)\right)(x, y)\right)-$

$f\left(x, y,\left(h\left(x_{p}\right)\right)(x, y)\right) \|+$

$\| \int_{0}^{x} \int_{0}^{y} K\left(x, y, s, t, u_{n}(s, t)\right) d s d t-$

$\int_{0}^{x} \int_{0}^{y} K\left(x, y, s, t, x_{p}(s, t)\right) d s d t \|$

$\leq L_{f}\left\|\left(h\left(u_{n}\right)\right)(x, y)-\left(h\left(x_{p}\right)\right)(x, y)\right\|+$

$\int_{0}^{x} \int_{0}^{y} \| K\left(x, y, s, t, u_{n}(s, t)\right)-$

$K\left(x, y, s, t, x_{p}(s, t)\right) \| d s d t$

$\leq L_{f} L_{h}\left\|u_{n}-x_{p}\right\|_{\tau} e^{\tau(x+y)}+$

$\int_{0}^{x} \int_{0}^{y} L_{K}(x, y, s, t)\left\|u_{n}(s, t)-x_{p}(s, t)\right\| d s d t$

$\leq L_{f} L_{h}\left\|u_{n}-x_{p}\right\|_{\tau} e^{\tau(x+y)}+\| u_{n}-$

$x_{p} \|_{\tau} \int_{0}^{x} \int_{0}^{y} L_{K}(x, y, s, t) e^{\tau(s+t)} d s d t$

$\leq L_{f} L_{h}\left\|u_{n}-x_{p}\right\|_{\tau} e^{\tau(x+y)}+L\left\|u_{n}-x_{p}\right\|_{\tau} e^{\tau(x+y)}$

$\leq\left(L_{f} L_{h}+L\right)\left\|u_{n}-x_{p}\right\|_{\tau} e^{\tau(x+y)}$

and we have

$\left\|x_{n+1}-x_{p}\right\|_{\tau} \leq\left(L_{f} L_{h}+L\right)\left\|u_{n}-x_{p}\right\|_{\tau}$

and 
$\left\|T x_{n}(x, y)-T x_{p}(x, y)\right\|=$

$$
\text { If } f\left(x, y,\left(h\left(x_{n}\right)\right)(x, y)\right)
$$

$+\int_{0}^{x} \int_{0}^{y} K\left(x, y, s, t, x_{n}(s, t)\right) d s d t$

$-f\left(x, y,\left(h\left(x_{p}\right)\right)(x, y)\right)$

$\left\|-\int_{0}^{x} \int_{0}^{y} K\left(x, y, s, t, x_{p}(s, t)\right) d s d t\right\|$

$\leq\left\|f\left(x, y,\left(h\left(x_{n}\right)\right)(x, y)\right)-f\left(x, y,\left(h\left(x_{p}\right)\right)(x, y)\right)\right\|+$

$\| \int_{0}^{x} \int_{0}^{y} K\left(x, y, s, t, x_{n}(s, t)\right) d s d t-$

$\int_{0}^{x} \int_{0}^{y} K\left(x, y, s, t, x_{p}(s, t)\right) d s d t \|$

$\leq L_{f}\left\|\left(h\left(x_{n}\right)\right)(x, y)-\left(h\left(x_{p}\right)\right)(x, y)\right\|+$

$\int_{0}^{x} \int_{0}^{y} \| K\left(x, y, s, t, x_{n}(s, t)\right)-$

$K\left(x, y, s, t, x_{p}(s, t)\right) \| d s d t$

$\leq L_{f} L_{h}\left\|x_{n}-x_{p}\right\|_{\tau} e^{\tau(x+y)}+$

$\int_{0}^{x} \int_{0}^{y} L_{K}(x, y, s, t)\left\|x_{n}(s, t)-x_{p}(s, t)\right\| d s d t$

$\leq L_{f} L_{h}\left\|x_{n}-x_{p}\right\|_{\tau} e^{\tau(x+y)}$

$+L\left\|x_{n}-x_{p}\right\|_{\tau} e^{\tau(x+y)}$

$\leq\left(L_{f} L_{h}+L\right)\left\|x_{n}-x_{p}\right\|_{\tau} e^{\tau(x+y)}$

and we obtain

$\left\|T x_{n}-T x_{p}\right\|_{\tau} \leq\left(L_{f} L_{h}+L\right)\left\|x_{n}-x_{p}\right\|_{\tau}$.

Similarly,

$\left\|T y_{n}-T x_{p}\right\|_{\tau}=\sup _{x, y \in \mathbb{R}_{+}}\left(\| T y_{n}(x, y)-\right.$

$\left.T x_{p}(x, y) \| e^{-\tau(x+y)}\right)$

and we have

$\left\|T y_{n}-T x_{p}\right\|_{\tau}=\left(L_{f} L_{h}+L\right)\left\|y_{n}-x_{p}\right\|_{\tau}$

(2.5)

and similarly,

$\left\|y_{n}-x_{p}\right\|_{\tau}=\sup _{x, y \in \mathbb{R}_{+}}\left(\| y_{n}(x, y)-\right.$

$\left.x_{p}(x, y) \| e^{-\tau(x+y)}\right)$

and

$\left\|y_{n}(x, y)-x_{p}(x, y)\right\|=\left\|T v_{n}(x, y)-T x_{p}(x, y)\right\|=$

$f\left(x, y,\left(h\left(v_{n}\right)\right)(x, y)\right)$

$+\int_{0}^{x} \int_{0}^{y} K\left(x, y, s, t, v_{n}(s, t)\right) d s d t$

$-f\left(x, y,\left(h\left(x_{p}\right)\right)(x, y)\right)$

$\left\|-\int_{0}^{x} \int_{0}^{y} K\left(x, y, s, t, x_{p}(s, t)\right) d s d t\right\|$

$\leq\left\|f\left(x, y,\left(h\left(v_{n}\right)\right)(x, y)\right)-f\left(x, y,\left(h\left(x_{p}\right)\right)(x, y)\right)\right\|+$

$\| \int_{0}^{x} \int_{0}^{y} K\left(x, y, s, t, v_{n}(s, t)\right) d s d t-$

$\int_{0}^{x} \int_{0}^{y} K\left(x, y, s, t, x_{p}(s, t)\right) d s d t \|$ $\leq L_{f}\left\|\left(h\left(v_{n}\right)\right)(x, y)-\left(h\left(x_{p}\right)\right)(x, y)\right\|+$

$\int_{0}^{x} \int_{0}^{y} \| K\left(x, y, s, t, v_{n}(s, t)\right)-$

$K\left(x, y, s, t, x_{p}(s, t)\right) \| d s d t$

$\leq L_{f} L_{h}\left\|v_{n}-x_{p}\right\|_{\tau} e^{\tau(x+y)}+$

$\int_{0}^{x} \int_{0}^{y} L_{K}(x, y, s, t)\left\|v_{n}(s, t)-x_{p}(s, t)\right\| d s d t$

$\leq L_{f} L_{h}\left\|v_{n}-x_{p}\right\|_{\tau} e^{\tau(x+y)}+L\left\|v_{n}-x_{p}\right\|_{\tau} e^{\tau(x+y)}$

$\leq\left(L_{f} L_{h}+L\right)\left\|v_{n}-x_{p}\right\|_{\tau} e^{\tau(x+y)}$.

Then, we have

$\left\|y_{n}-x_{p}\right\|_{\tau} \leq\left(L_{f} L_{h}+L\right)\left\|v_{n}-x_{p}\right\|_{\tau}$.

(2.6)

By using (2.4), we obtain

$\left\|v_{n}-x_{p}\right\|_{\tau}=\left\|\frac{\left(1-\beta_{n}\right)}{k} x_{n}+\left(1-\frac{\left(1-\beta_{n}\right)}{k}\right) T x_{n}-x_{p}\right\|_{\tau}$

$\leq \frac{\left(1-\beta_{n}\right)}{k}\left\|x_{n}-x_{p}\right\|_{\tau}+\left(1-\frac{\left(1-\beta_{n}\right)}{k}\right)\left\|T x_{n}-x_{p}\right\|_{\tau}$

$\leq \frac{\left(1-\beta_{n}\right)}{k}\left\|x_{n}-x_{p}\right\|_{\tau}+\left(L_{f} L_{h}+L\right)\left(1-\frac{\left(1-\beta_{n}\right)}{k}\right) \| x_{n}-$

$x_{p}\left\|_{\tau} \leq\right\| x_{n}-x_{p} \|_{\tau}$

and combining (2.4), (2.5), (2.6), and (2.7)

$\left\|u_{n}-x_{p}\right\|_{\tau}=\left\|\frac{\left(1-\alpha_{n}\right)}{k} T x_{n}+\left(1-\frac{\left(1-\alpha_{n}\right)}{k}\right) T y_{n}-x_{p}\right\|_{\tau}$

$\leq \frac{\left(1-\alpha_{n}\right)}{k}\left\|T x_{n}-x_{p}\right\|_{\tau}+\left(1-\frac{\left(1-\alpha_{n}\right)}{k}\right)\left\|T y_{n}-x_{p}\right\|_{\tau}$

$\leq \frac{\left(1-\alpha_{n}\right)}{k}\left(L_{f} L_{h}+L\right)\left\|x_{n}-x_{p}\right\|_{\tau}+\left(L_{f} L_{h}+\right.$

L) $\left(1-\frac{\left(1-\alpha_{n}\right)}{k}\right)\left\|y_{n}-x_{p}\right\|_{\tau}$

$\leq \frac{\left(1-\alpha_{n}\right)}{k}\left(L_{f} L_{h}+L\right)\left\|x_{n}-x_{p}\right\|_{\tau}+\left(L_{f} L_{h}+L\right)^{2}(1-$

$\left.\frac{\left(1-\alpha_{n}\right)}{k}\right)\left\|v_{n}-x_{p}\right\|_{\tau}$

$\leq \frac{\left(1-\alpha_{n}\right)}{k}\left(L_{f} L_{h}+L\right)\left\|x_{n}-x_{p}\right\|_{\tau}+\left(L_{f} L_{h}+L\right)^{2}(1-$

$\left.\frac{\left(1-\alpha_{n}\right)}{k}\right)\left\|x_{n}-x_{p}\right\|_{\tau}$

$\leq\left(L_{f} L_{h}+L\right)\left\|x_{n}-x_{p}\right\|_{\tau}$

and we have

$\left\|x_{n+1}-x_{p}\right\|_{\tau} \leq\left\|T u_{n}-x_{p}\right\|_{\tau}$

$\leq\left(L_{f} L_{h}+L\right)\left\|u_{n}-x_{p}\right\|_{\tau}$

$\leq\left(L_{f} L_{h}+L\right)^{2}\left\|x_{n}-x_{p}\right\|_{\tau}$

by induction we obtain

$\left\|x_{n+1}-x_{p}\right\|_{\tau} \leq\left(L_{f} L_{h}+L\right)^{2 n}\left\|x_{0}-x_{p}\right\|_{\tau}$

Taking the limit on both sides of (2.9) and using

$\left(L_{f} L_{h}+L\right)<1$, we obtain

$\lim _{n \rightarrow \infty}\left\|x_{n}-x_{p}\right\|_{\tau}=0$.

We consider the equation (1.1), we have 
$S\left(w_{n}(x, y)\right)=f_{1}\left(x, y,\left(h\left(w_{n}\right)\right)(x, y)\right)+$ $\int_{0}^{x} \int_{0}^{y} K_{1}\left(x, y, s, t, w_{n}(s, t)\right) d s d t \quad x, y \in \mathbb{R}_{+}$.

We have

\section{Teorem 2.2 We suppose that}

i) $f, K, f_{1}, K_{1}, h$ satisfy conditions c1-c6 in Theorem 1.1

ii) there exits $\varepsilon_{1}>0$ such that

$$
\left\|f(x, y, w)-f_{1}(x, y, w)\right\|_{\tau} \leq \varepsilon_{1}
$$

for all $x, y \in \mathbb{R}_{+}, \mathrm{w} \in E$;

iii) there exits $\varepsilon_{2}>0$ such that

$$
\left\|K(x, y, s, t, w)-K_{1}(x, y, s, t, w)\right\|_{\tau} \leq L \varepsilon_{2}
$$

for all $x, y, s, t \in \mathbb{R}_{+}, \forall w \in E$;

iv) $\left\{w_{n}\right\}_{n=1}^{\infty}$ be an iterative sequence generated by

$$
\left\{\begin{array}{c}
w_{n+1}=S \eta_{n} \\
\eta_{n}=\frac{\left(1-\alpha_{n}\right)}{k} S w_{n}+\left(1-\frac{\left(1-\alpha_{n}\right)}{k}\right) S \mu_{n} \\
\mu_{n}=S \zeta_{n} \\
\zeta_{n}=\frac{\left(1-\beta_{n}\right)}{k} w_{n}+\left(1-\frac{\left(1-\beta_{n}\right)}{k}\right) S w_{n} .
\end{array}\right.
$$

Then

a) the equations (1.6) and (2.10) have a unique solution $x_{p}, w_{p}$ respectively;

b) $\quad\left\|w_{p}-x_{p}\right\| \leq\left[\frac{1+\left(L_{f} L_{h}+L\right)+\left(L_{f} L_{h}+L\right)^{2}+\left(L_{f} L_{h}+L\right)^{3}}{1-\left(L_{f} L_{h}+L\right)^{2}}\right]\left(\varepsilon_{1}+\right.$ $\left.L \varepsilon_{2}\right)$.

Proof The assumptions (c1)-(c6) of Theorem 1.1, we obtain

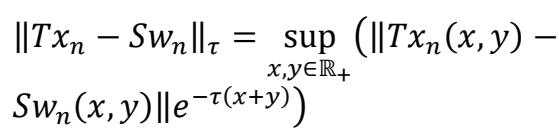

and

$$
\begin{aligned}
& \left\|T x_{n}(x, y)-S w_{n}(x, y)\right\| \leq \\
& \left\|\begin{array}{c}
f\left(x, y,\left(h\left(x_{n}\right)\right)(x, y)\right) \\
+\int_{0}^{x} \int_{0}^{y} K\left(x, y, s, t, x_{n}(s, t)\right) d s d t \\
-f_{1}\left(x, y,\left(h\left(w_{n}\right)\right)(x, y)\right) \\
-\int_{0}^{x} \int_{0}^{y} K_{1}\left(x, y, s, t, w_{n}(s, t)\right) d s d t
\end{array}\right\| \\
& \leq\left\|f\left(x, y,\left(h\left(x_{n}\right)\right)(x, y)\right)-f\left(x, y,\left(h\left(w_{n}\right)\right)(x, y)\right)\right\|+ \\
& \left\|f\left(x, y, h\left(w_{n}(x, y)\right)\right)-f_{1}\left(x, y,\left(h\left(w_{n}\right)\right)(x, y)\right)\right\|+
\end{aligned}
$$

$\| \int_{0}^{x} \int_{0}^{y} K\left(x, y, s, t, x_{n}(s, t)\right) d s d t-$ $\int_{0}^{x} \int_{0}^{y} K\left(x, y, s, t, w_{n}(s, t)\right) d s d t \|+$ $\| \int_{0}^{x} \int_{0}^{y} K\left(x, y, s, t, w_{n}(s, t)\right) d s d t-$ $\int_{0}^{x} \int_{0}^{y} K_{1}\left(x, y, s, t, w_{n}(s, t)\right) d s d t \|$ $\leq L_{f}\left\|\left(h\left(x_{n}\right)\right)(x, y)-\left(h\left(w_{n}\right)\right)(x, y)\right\|+\varepsilon_{1} e^{\tau(x+y)}+$ $\int_{0}^{x} \int_{0}^{y} L_{K}(x, y, s, t)\left\|x_{n}(s, t)-w_{n}(s, t)\right\| d s d t+$ $L \varepsilon_{2} e^{\tau(x+y)}$

$\leq L_{f} L_{h}\left\|x_{n}-w_{n}\right\|_{\tau} e^{\tau(x+y)}+\varepsilon_{1} e^{\tau(x+y)}+L \| x_{n}-$ $w_{n} \|_{\tau} e^{\tau(x+y)}+L \varepsilon_{2} e^{\tau(x+y)}$

$\leq\left[\left(L_{f} L_{h}+L\right)\left\|x_{n}-w_{n}\right\|_{\tau}+\left(\varepsilon_{1}+L \varepsilon_{2}\right)\right] e^{\tau(x+y)}$

By using (2.11), we have

$\left\|T x_{n}-S w_{n}\right\|_{\tau} \leq\left(L_{f} L_{h}+L\right)\left\|x_{n}-w_{n}\right\|_{\tau}+\varepsilon_{1}+L \varepsilon_{2}$

and we obtain

$\left\|v_{n}-\zeta_{n}\right\|_{\tau} \leq \| \frac{\left(1-\beta_{n}\right)}{k} x_{n}+\left(1-\frac{\left(1-\beta_{n}\right)}{k}\right) T x_{n}-$ $\frac{\left(1-\beta_{n}\right)}{k} w_{n}-\left(1-\frac{\left(1-\beta_{n}\right)}{k}\right) S w_{n} \|$ $\leq \frac{\left(1-\beta_{n}\right)}{k}\left\|x_{n}-w_{n}\right\|_{\tau}+\left(1-\frac{\left(1-\beta_{n}\right)}{k}\right)\left\|T x_{n}-S w_{n}\right\|_{\tau}$ $\leq \frac{\left(1-\beta_{n}\right)}{k}\left\|x_{n}-w_{n}\right\|_{\tau}+\left(1-\frac{\left(1-\beta_{n}\right)}{k}\right)\left(L_{f} L_{h}+\right.$ $L)\left\|x_{n}-w_{n}\right\|_{\tau}+\left(1-\frac{\left(1-\beta_{n}\right)}{k}\right)\left(\varepsilon_{1}+L \varepsilon_{2}\right)$ $\leq\left\|x_{n}-w_{n}\right\|_{\tau}+\left(1-\frac{\left(1-\beta_{n}\right)}{k}\right)\left(\varepsilon_{1}+L \varepsilon_{2}\right)$.

Similarly to (2.11),

$\left\|y_{n}-\mu_{n}\right\|=\left\|T v_{n}-S \zeta_{n}\right\| \leq\left[\left(L_{f} L_{h}+L\right) \| v_{n}-\right.$ $\left.\zeta_{n} \|_{\tau}+\left(\varepsilon_{1}+L \varepsilon_{2}\right)\right] e^{\tau(x+y)}$.

Thus, we have

$\left\|y_{n}-\mu_{n}\right\|_{\tau}=\left\|T v_{n}-S \zeta_{n}\right\|_{\tau} \leq\left(L_{f} L_{h}+L\right) \| v_{n}-$

$\zeta_{n} \|_{\tau}+\left(\varepsilon_{1}+L \varepsilon_{2}\right)$

and

$\left\|T y_{n}-S \mu_{n}\right\|_{\tau}=\sup _{x, y \in \mathbb{R}_{+}}\left(\| T y_{n}(x, y)-\right.$

$\left.S \mu_{n}(x, y) \| e^{-\tau(x+y)}\right)$

and

$\left\|T y_{n}-S \mu_{n}\right\| \leq \| f\left(x, y, h\left(y_{n}(x, y)\right)\right)-$

$f\left(x, y,\left(h\left(\mu_{n}\right)\right)(x, y)\right)\|+\| f\left(x, y,\left(h\left(\mu_{n}\right)\right)(x, y)\right)-$

$f_{1}\left(x, y, h\left(h\left(\mu_{n}\right)\right)(x, y)\right) \|+$

$\| \int_{0}^{x} \int_{0}^{y} K\left(x, y, s, t, y_{n}(s, t)\right) d s d t-$ $\int_{0}^{x} \int_{0}^{y} K\left(x, y, s, t, \mu_{n}(s, t)\right) d s d t \|+$ $\| \int_{0}^{x} \int_{0}^{y} K\left(x, y, s, t, \mu_{n}(s, t)\right) d s d t-$ $\int_{0}^{x} \int_{0}^{y} K_{1}\left(x, y, s, t, \mu_{n}(s, t)\right) d s d t \|$ 
$\leq L_{f}\left\|\left(h\left(y_{n}\right)\right)(x, y)-\left(h\left(\mu_{n}\right)\right)(x, y)\right\|+\varepsilon_{1} e^{\tau(x+y)}+$ $\int_{0}^{x} \int_{0}^{y} L_{K}(x, y, s, t)\left\|y_{n}(s, t)-\mu_{n}(s, t)\right\| d s d t+$ $L \varepsilon_{2} e^{\tau(x+y)}$

$\leq\left[\left(L_{f} L_{h}+L\right)\left\|y_{n}-\mu_{n}\right\|_{\tau}+\left(\varepsilon_{1}+L \varepsilon_{2}\right)\right] e^{\tau(x+y)}$.

By using (2.15), we have

$\left\|T y_{n}-S \mu_{n}\right\|_{\tau} \leq\left(L_{f} L_{h}+L\right)\left\|y_{n}-\mu_{n}\right\|_{\tau}+\varepsilon_{1}+L \varepsilon_{2}$.

Combining (2.12), (2.13), (2.14), and (2.16), we have

$\left\|\eta_{n}-u_{n}\right\|_{\tau} \leq \frac{\left(1-\alpha_{n}\right)}{k}\left\|T x_{n}-S w_{n}\right\|_{\tau}+(1-$

$\left.\frac{\left(1-\alpha_{n}\right)}{k}\right)\left\|T y_{n}-S \mu_{n}\right\|_{\tau}$

$\leq \frac{\left(1-\alpha_{n}\right)}{k}\left[\left(L_{f} L_{h}+L\right)\left\|x_{n}-w_{n}\right\|_{\tau}+\varepsilon_{1}+L \varepsilon_{2}\right]+$ $\left(1-\frac{\left(1-\alpha_{n}\right)}{k}\right)\left[\left(L_{f} L_{h}+L\right)\left\|y_{n}-\mu_{n}\right\|_{\tau}+\varepsilon_{1}+L \varepsilon_{2}\right]$ $\leq \frac{\left(1-\alpha_{n}\right)}{k}\left(L_{f} L_{h}+L\right)\left\|x_{n}-w_{n}\right\|_{\tau}+\frac{\left(1-\alpha_{n}\right)}{k} \varepsilon_{1}+$ $L \frac{\left(1-\alpha_{n}\right)}{k} \varepsilon_{2}+\left(1-\frac{\left(1-\alpha_{n}\right)}{k}\right)\left(L_{f} L_{h}+L\right)\left\|y_{n}-\mu_{n}\right\|_{\tau}+$ $\left(1-\frac{\left(1-\alpha_{n}\right)}{k}\right) \varepsilon_{1}+L\left(1-\frac{\left(1-\alpha_{n}\right)}{k}\right) \varepsilon_{2}$ $\leq \frac{\left(1-\alpha_{n}\right)}{k}\left(L_{f} L_{h}+L\right)\left\|x_{n}-w_{n}\right\|_{\tau}+\varepsilon_{1}+L \varepsilon_{2}+$ $\left(1-\frac{\left(1-\alpha_{n}\right)}{k}\right)\left(L_{f} L_{h}+L\right)\left\|y_{n}-\mu_{n}\right\|_{\tau}$ $\leq \frac{\left(1-\alpha_{n}\right)}{k}\left(L_{f} L_{h}+L\right)\left\|x_{n}-w_{n}\right\|_{\tau}+\varepsilon_{1}+L \varepsilon_{2}+$ $\left(1-\frac{\left(1-\alpha_{n}\right)}{k}\right)\left(L_{f} L_{h}+L\right)\left[\left(L_{f} L_{h}+L\right)\left\|v_{n}-\zeta_{n}\right\|_{\tau}+\right.$ $\left.\varepsilon_{1}+L \varepsilon_{2}\right]$ $\leq \frac{\left(1-\alpha_{n}\right)}{k}\left(L_{f} L_{h}+L\right)\left\|x_{n}-w_{n}\right\|_{\tau}+\varepsilon_{1}+L \varepsilon_{2}+$ $\left(1-\frac{\left(1-\alpha_{n}\right)}{k}\right)\left(L_{f} L_{h}+L\right)^{2}\left\|v_{n}-\zeta_{n}\right\|_{\tau}+\left(L_{f} L_{h}+\right.$ L) $\left(1-\frac{\left(1-\alpha_{n}\right)}{k}\right) \varepsilon_{1}+L\left(1-\frac{\left(1-\alpha_{n}\right)}{k}\right)\left(L_{f} L_{h}+L\right) \varepsilon_{2}$ $\leq \frac{\left(1-\alpha_{n}\right)}{k}\left(L_{f} L_{h}+L\right)\left\|x_{n}-w_{n}\right\|_{\tau}+\varepsilon_{1}+L \varepsilon_{2}+$ $\left(1-\frac{\left(1-\alpha_{n}\right)}{k}\right)\left(L_{f} L_{h}+L\right)^{2}\left\|x_{n}-w_{n}\right\|_{\tau}+(1-$ $\left.\frac{\left(1-\alpha_{n}\right)}{k}\right)\left(1-\frac{\left(1-\beta_{n}\right)}{k}\right)\left(L_{f} L_{h}+L\right)^{2} \varepsilon_{1}+L(1-$ $\left.\frac{\left(1-\alpha_{n}\right)}{k}\right)\left(1-\frac{\left(1-\beta_{n}\right)}{k}\right)\left(L_{f} L_{h}+L\right)^{2} \varepsilon_{2}+(1-$ $\left.\frac{\left(1-\alpha_{n}\right)}{k}\right)\left(L_{f} L_{h}+L\right) \varepsilon_{1}+L\left(1-\frac{\left(1-\alpha_{n}\right)}{k}\right)\left(L_{f} L_{h}+L\right) \varepsilon_{2}$ $\leq\left(L_{f} L_{h}+L\right)\left\|x_{n}-w_{n}\right\|_{\tau}+\varepsilon_{1}+L \varepsilon_{2}+(1-$ $\left.\frac{\left(1-\alpha_{n}\right)}{k}\right)\left(1-\frac{\left(1-\beta_{n}\right)}{k}\right)\left(L_{f} L_{h}+L\right)^{2} \varepsilon_{1}+L(1-$ $\left.\frac{\left(1-\alpha_{n}\right)}{k}\right)\left(1-\frac{\left(1-\beta_{n}\right)}{k}\right)\left(L_{f} L_{h}+L\right)^{2} \varepsilon_{2}+(1-$ $\left.\frac{\left(1-\alpha_{n}\right)}{k}\right)\left(L_{f} L_{h}+L\right) \varepsilon_{1}+L\left(1-\frac{\left(1-\alpha_{n}\right)}{k}\right)\left(L_{f} L_{h}+L\right) \varepsilon_{2}$

and similarly

$\left\|x_{n+1}-w_{n+1}\right\|_{\tau} \leq\left\|T u_{n}-S \eta_{n}\right\|_{\tau} \leq\left(L_{f} L_{h}+\right.$ L) $\left\|u_{n}-\eta_{n}\right\|_{\tau}+\varepsilon_{1}+L \varepsilon_{2}$

$\leq\left(L_{f} L_{h}+L\right)^{2}\left\|x_{n}-w_{n}\right\|_{\tau}+\left(L_{f} L_{h}+L\right) \varepsilon_{1}+$ $L\left(L_{f} L_{h}+L\right) \varepsilon_{2}+\varepsilon_{1}+L \varepsilon_{2}+\left(1-\frac{\left(1-\alpha_{n}\right)}{k}\right)(1-$ $\left.\frac{\left(1-\beta_{n}\right)}{k}\right)\left(L_{f} L_{h}+L\right)^{3} \varepsilon_{1}+L\left(1-\frac{\left(1-\alpha_{n}\right)}{k}\right)(1-$
$\left.\frac{\left(1-\beta_{n}\right)}{k}\right)\left(L_{f} L_{h}+L\right)^{3} \varepsilon_{2}+\left(1-\frac{\left(1-\alpha_{n}\right)}{k}\right)\left(L_{f} L_{h}+\right.$

$L)^{2} \varepsilon_{1}+L\left(1-\frac{\left(1-\alpha_{n}\right)}{k}\right)\left(L_{f} L_{h}+L\right)^{2} \varepsilon_{2}$

and we get

$\left(L_{f} L_{h}+L\right)^{2}=(1-\theta)$

Then, we have

$\left\|x_{n+1}-w_{n+1}\right\| \leq(1-\theta)\left\|x_{n}-w_{n}\right\|+\theta\left[\frac{1+\left(L_{f} L_{h}+L\right)}{\theta}+\right.$

$\left.\frac{\left(L_{f} L_{h}+L\right)^{2}+\left(L_{f} L_{h}+L\right)^{3}}{\theta}\right]\left(\varepsilon_{1}+L \varepsilon_{2}\right)$.

Denote that

$\sigma_{n+1}=\left\|x_{n+1}-w_{n+1}\right\|$

$\sigma_{n}=\left\|x_{n}-w_{n}\right\|$

$\lambda_{n}=\theta=1-\left(L_{f} L_{h}+L\right)^{2}$

$m_{n}=\left[\frac{1+\left(L_{f} L_{h}+L\right)}{1-\left(L_{f} L_{h}+L\right)^{2}}+\frac{\left(L_{f} L_{h}+L\right)^{2}+\left(L_{f} L_{h}+L\right)^{3}}{1-\left(L_{f} L_{h}+L\right)^{2}}\right]\left(\varepsilon_{1}+L \varepsilon_{2}\right)$.

It can be seen that (2.17) satisfies all the conditions in Lemma 1.3, and hence it follows from its conclusion that

$\left\|x_{p}-w_{p}\right\|=\left[\frac{1+\left(L_{f} L_{h}+L\right)+\left(L_{f} L_{h}+L\right)^{2}+\left(L_{f} L_{h}+L\right)^{3}}{1-\left(L_{f} L_{h}+L\right)^{2}}\right]\left(\varepsilon_{1}+L \varepsilon_{2}\right)$.

\section{Conclusion}

In this work, we have shown that the iteration method (1.5) converges to the solution of the more general Volterra integral equation in two variables (1.1). Finally, we have proved a data dependence result can be obtained for the solution of the integral equation (1.1).

\section{Author's Contributions}

Samet Maldar compiled information from the literature and wrote the manuscript.

\section{Ethics}

There are no ethical issues after the publication of this manuscript.

\section{References}

1. Rawat, S., Dimri, R. C., and Bartwal, A. 2021. A New Iterative Scheme for Approximation of Fixed Points of Suzuki's Generalized Nonexpansive Mappings. doi 10.20944/preprints202105.0125.v1.

2. Akewe, H. 2016. The stability of a modified Jungck-Mann hybrid fixed point iteration procedure. International Journal of 
Mathematical Analysis and Optimization: Theory and Applications; 2016, 95-104.

3. Dogan, K. 2018. Daha Hizlı Mann Sabit Nokta Yinelemes Uzerine Bir Çalısma. Afyon Kocatepe Universitesi Fen ve Mühendislik Bilimleri Dergisi; 18(3), 852-860.

4. Başarır M and Şahin A. 2017. Some Results of The New Iterative Scheme in Hyperbolic Space. Communications of the Korean Mathematical Society; 32 (4), 1009-1024.

5. Gürsoy, F., Karakaya, V., and Rhoades, B. E. 2014, January. Some convergence and stability results for the Kirk multistep and Kirk-SP fixed point iterative algorithms. In Abstract and Applied Analysis; 2014, 1-12.

6. Lungu, N. ve Rus, I. A. 2009. On a Functional Volterra-Fredholm Integral Equation via Picard Operator. Journal of Mathematical Inequalities; 3 (4), 519-527.

7. Bielecki, A. 1956. Un Remarque sur L'application de la Méthode de Banach-Cacciopoli-Tikhonov dans la Théorie de L'equation s= f (x, y, z, p, q). Bull. Acad. Polon. Sci. Sér. Sci. Math. Phys. Astr; 4, 265-268.

8. Banas, J., and Lecko, M. 2002. Fixed points of the product of operators in Banach algebra. Panamerican Mathematical Journal; 12(2), 101-109.

9. Hadizadeh, M., and Asgary, M. 2005. An efficient numerical aproximation for the linear class of mixed integral equations. Applied mathematics and computation; 167(2), 10901100.

10. Çakan, Ü., and Özdemir, İ. 2015. An application of Darbo fixedpoint theorem to a class of functional integral equations. Numerical Functional Analysis and Optimization; 36(1), 29-40.

11. Cakan, Ü., and Özdemir, İ. 2017. Applications of measure of noncompactness and Darbo's fixed point theorem to nonlinear integral equations in Banach spaces. Numerical Functional Analysis and Optimization; 38(5), 641-673.

12. Cakan, Ü. 2017. On monotonic solutions of some nonlinear fractional integral equations. Nonlinear Funct. Anal. Appl.; 22(2), 259-273.

13. Dobritoiu M, 2008. System of Integral Equations with Modified Argument. Carpathian J. Math, 24(2): 26-36.

14. Craciun, C., and Serban, M. A. 2011. A nonlinear integral equation via Picard operators. Fixed point theory; 12(1), 57-70.

15. Gursoy, F. 2014. Applications of normal S-iterative method to a nonlinear integral equation. The Scientific World Journal; 2014, $1-5$.

16. Atalan, Y., and Karakaya, V. 2017. Stability of nonlinear Volterra-Fredholm integro differential equation: A fixed point approach. Creat. Math. Inform. 26 (2017), No. 3, 247-254.

17. Atalan, Y., and Karakaya, V. 2017. Iterative Solution of Functional Volterra-Fredholm Integral Equation with Deviating Argument. J. Nonlinear and Convex Analysis; 18(4), 675-684.

18. Karapınar, E., Kumari, P. S., and Lateef, D. 2018. A new approach to the solution of the Fredholm integral equation via a fixed point on extended b-metric spaces. Symmetry; 10(10), 512

19. Garodia, C., and Uddin, I. 2018. Solution of a nonlinear integra equation via new fixed point iteration process. arXiv preprin arXiv:1809.03771
20. Abbas, M., Ibrahim, Y., Khan, A. R., and De la Sen, M. 2019. Strong Convergence of a System of Generalized Mixed Equilibrium Problem, Split Variational Inclusion Problem and Fixed-Point Problem in Banach Spaces. Symmetry, 11(5), 722

21. Atalan, Y. 2019. İteratif Yaklaşım Altında Bir Fonksiyonelİntegral Denklem Sinıfının Cözümünün İncelenmesi. I ğdır Üniversitesi Fen Bilimleri Enstitüsü Dergisi; 9(3), 1622-1632.

22. Ilea, V., and Otrocol, D. 2020. Existence and Uniqueness of the Solution for an Integral Equation with Supremum, via wDistances. Symmetry; 12(9), 1554

23. Ciplea, S. A., Lungu, N., Marian, D., and Rassias, T. M. 2020. On Hyers-Ulam-Rassias stability of a Volterra-Hammerstein functional integral equation. arXiv preprint arXiv:2001.07760.

24. Abdou, M. A., Soliman, A. A., and Abdel-Aty, M. A. 2020. On a discussion of Volterra-Fredholm integral equation with discontinuous kernel. Journal of the Egyptian Mathematical Society; 28(1), 1-10.

25. Beiglo, H., and Gachpazan, M. 2020. Numerical solution of nonlinear mixed Volterra-Fredholm integral equations in complex plane via PQWs. Applied Mathematics and Computation; 369, 124828, 1-9.

26. Panda, S. K., Abdeljawad, T., \& Ravichandran, C. 2020. Novel fixed-point approach to Atangana-Baleanu fractional and LpFredholm integral equations. Alexandria Engineering Journal; $59,4,1959-1970$

27. Okeke, G. A., \& Abbas, M. 2020. Fejér monotonicity and fixedpoint theorems with applications to a nonlinear integral equation in complex valued Banach spaces. Applied General Topology; 21(1), 135-158

28. Gutiérrez, J. M., Hernández-Verón, M. Á., and Martínez, E. 2020. Improved Iterative Solution of Linear Fredholm Integral Equations of Second Kind via Inverse-Free Iterative Schemes. Mathematics; 8 (10), 1747.

29. Hacioğlu, E., Gürsoy, F., Maldar, S., Atalan, Y., and Milovanović, G. V. 2021. Iterative approximation of fixed points and applications to two-point second-order boundary value problems and to machine learning. Applied Numerical Mathematics; 167, 143-172.

30. Maldar, S. 2020. Yeni Bir İterasyon Yöntemi İçin Yakınsaklık Hızı. Ĭgdır Üniversitesi Fen Bilimleri Enstitüsü Dergisi; 10(2), 1263-1272.

31. Soltuz S. M. and Grosan T. 2008. Data dependence for Ishikawa iteration when dealing with contractive like operators. Fixed Point Theory Appl.; 2008 (2008), 1-7. 